\title{
Propuesta didáctica para la enseñanza de la parábola en Ingeniería Civil
}

\author{
Sara Giraldo J. \\ Frey Rodríguez $P$.
}

Recibido el 10 de junio de 2009. Aprobado el 19 de octubre de 2009

\begin{abstract}
Resumen
Este artículo da cuenta de una experiencia en el aula de clase para la enseñanza de la parábola en estudiantes de Ingeniería Civil de la Corporación Universitaria Minuto de Dios (UNIMINUTO) desarrollada durante el segundo semestre de 2004. En su diseño, se consideraron referentes pedagógicos y matemáticos que sirvieron como derroteros para la construcción de una propuesta metodológica donde el estudiante se enfrenta a situaciones muy similares a las que desarrollará en su vida profesional. Para la implementación se tuvieron en cuenta tres fases: contextualización, construcción y ampliación. Finalmente se presentan los resultados de la puesta en el aula desde tres aspectos: el trabajo de los estudiantes, la comprensión y la interacción didáctica, con las cuales se evidenció que para introducir la parábola a estudiantes de Ingeniería Civil debe iniciarse con una situación problema, específica de su futuro campo de acción, ya que de esta manera se motiva y se destaca la importancia de dicha cónica.
\end{abstract}

\section{Palabras clave:}

Parábola, Representaciones, Situación problema, Matemáticas, Pedagogía y Didáctica.

\begin{abstract}
This article describes an experience of classroom teaching in the parable of civil engineering students the Corporación Universitaria Minuto de Dios (UNIMINUTO) developed during the second half of 2004. In their design, were considered related to teaching math and served as paths for the construction of a methodology where the student is faced with situations similar to those that live in their future careers. For implementation were taken into account three phases: context, construction and expansion. Finally presents the results of placing in the classroom from three aspects: students' work, understanding and teaching interaction, with which it was shown that the parable to introduce civil engineering students should start with a problem situation, specific to their future field of action, as this is encouraged, and emphasizes the importance of this cone.
\end{abstract}

\section{Keywords}

Parable, Offices, Location problem, Mathematics, Pedagogy, Didactic. 


\section{Introducción}

Tradicionalmente la enseñanza de las cónicas se realiza de forma netamente algebraica, dejando de lado, sus características como lugares geométricos' (Sánchez, 1996). Muchas disciplinas, entre ellas la ingeniería, requieren que sus profesionales se apropien de dichos objetos matemáticos de una manera aplicada a partir de la identificación de aspectos relevantes dentro de un contexto específico.

Por medio de este artículo, se pretende compartir los resultados de una experiencia de aula desarrollada durante el segundo semestre de 2004 en UNIMINUTO, que tenía como objetivo dar respuesta a la pregunta ¿Cómo introducir el estudio de la parábola de tal forma que los estudiantes puedan establecer vínculos entre el acercamiento realizado y su futuro desempeño laboral?

Esta investigación fue enmarcada como un estudio de caso, ya que la intención era indagar por la manera en que se debía abordar el estudio de la parábola con estudiantes con carencias en comprensión lectora, fuertes dificultades matemáticas y poca o ninguna experiencia con la geometría en su formación secundaria. (UNIMINUTO, 2001).

Inicialmente se presenta el soporte pedagógico y matemático de la intervención, luego, se mencionan los aspectos considerados en el diseño global de la propuesta y la manera como se realizó su registro; por último, a partir de los resultados obtenidos durante tres sesiones de trabajo en el aula, se evidenció la pertinencia de la experiencia partiendo de una situación problema, pasando por un descubrimiento dirigido o construcción de la parábola y finalizando con aplicaciones a la Ingeniería Civil.

\section{Metodología Marco referencial pedagógico.}

Desde el punto de vista didáctico, la enseñanza de la parábola se puede abordar mediante dos aproximaciones: el pragmático y el intelectual. En el primero de ellos, se sugiere organizar la enseñanza a partir de la capacidad de observar y construir, teniendo siempre en cuenta las razones personales que dan los estudiantes. El acercamiento intelectual o teórico se apoya en la formulación de propiedades de un objeto y sus relaciones.

\footnotetext{
' Se entiende por lugar geométrico como el conjunto de puntos del plano que cumplen determinada característica común. Si la característica se puede representar mediante una relación algebraica, a dicha relación se le llama ecuación del lugar geométrico (Joya, 2007)
}

Desde una mirada pragmática, la propuesta más pertinente es la de Sánchez (1996), quien plantea, desde la teoría de las situaciones didácticas de Guy Brousseau y el aprendizaje significativo de Ausbel, un método constructivo para la enseñanza de cualquier tema en matemáticas, con el que se pretende que el estudiante construya aprendizajes significativos modificando sus esquemas de conocimiento. Para tal fin, los principios didácticos que orientan este enfoque son: el diseño de actividades que contribuyan a que la nueva información se conecte con los pre-saberes, el incentivo y la mayor atención a los estudiantes, una metodología soportada en el permanente diálogo entre pares y el uso de situaciones problema como camino para cualquier conocimiento nuevo.

Otros investigadores proponen acercamientos constructivistas a partir de diferentes herramientas. Hansen (2001) recomienda presentar modelos reales a los estudiantes de las formas geométricas asociadas a las superficies cónicas de manera que las puedan tocar y sentir. Bruño (1960), sugiere para la enseñanza de las cónicas las construcciones con regla y compás. Y el Ministerio de Educación Nacional (2004), promueve el uso de las nuevas herramientas tecnológicas, específicamente los programas de geometría dinámica, que permiten explorar diversas posibilidades a partir de elementos como el arrastre de las figuras que favorecen la búsqueda de rasgos que permanecen "fijos o invariantes" durante la deformación de los mismos, el uso extensivo del lugar geométrico como la huella que deja una figura cuando se arrastra y la animación de figuras que permite presenciar el proceso constructivo de un hecho geométrico.

Desde lo intelectual o teórico, investigadores como Gómez y Carulla (1998), Janvier (1987) y Font (2001) señalan que en la enseñanza de diferentes objetos matemáticos, como la parábola, juegan un papel importante las actividades en las que el estudiante debe pasar de una representación a otra; al respecto, específicamente Janvier (1987) afirma "la naturaleza de las representaciones ${ }^{2}$ matemáticas ostensivas $^{3}$ influye en el tipo de comprensión generada en el alumno; y, recíprocamente, el tipo de comprensión del alumno determina el tipo de representación ostensiva a utilizar".

\footnotetext{
2 "puede ser una combinación de algo escrito en el papel, algo que existe bajo la forma de objetos físicos y de un arreglo cuidadosamente construido de la idea en su mente" (Janvier, citado por Font, 2001)

${ }^{3}$ Janvier utiliza el término ostensivo en el sentido de que se puede mostrar a otro directamente. Por representación ostensiva entiendo, por ejemplo, la fórmula de la función que el profesor escribe en la pizarra y el alumno ve directamente. (citado por Font, 2001)
} 
Gómez y Carulla (1998) mencionan las representaciones más comunes de la parábola:

-Simbólica, con un lenguaje propio del campo algebraico.

-Gráfica, donde se hace referencia a la representación en el plano cartesiano.

Geométrica, que aunque es de alguna manera gráfica en ella no se utiliza un sistema de referencia cartesiano.

Numérica, en donde se habla de los valores numéricos de la función cuadrática representados de diferentes formas.

-Verbal, en la que se describen situaciones del mundo real y del mundo de las matemáticas que pueden ser modeladas en cualquiera de los otros sistemas de representación.

\section{Marco referencial matemático}

Desde el punto de vista matemático, la parábola es abordada en los textos universitarios desde los siguientes enfoques: como lugar geométrico, función polinómica y función de proporción.

Como lugar geométrico se consideran dos tipos de acercamiento: el geométrico y el algebraico. En el primero de ellos se considera la parábola desde una noción estática, para Bruño (1960) es "...una curva plana no cerrada, con una sola rama que se extiende indefinidamente y en la cual cada punto equidista de un punto fijo y una recta fija dados en un plano" y desde una dinámica, Lehman (1996) la considera como "... el lugar geométrico de un punto que se mueve en el plano de tal manera que su distancia desde una recta fija situada en el plano, es siempre igual a su distancia a un punto fijo del plano y que no pertenece a la recta". Algebraicamente es la representación gráfica de la igualdad de expresiones verbales como son la distancia de un punto al foco y distancia de punto a recta. (Stewart, 2001).

Como función polinómica, la parábola es una función de la forma $f(x)=a x^{2}+b x+c$ donde $a, b, c$ son números reales con $a \neq 0$ o en su forma general

$A x^{2}+C x+D y+F=0 \quad$ Bajo este acercamiento solamente se hace reterencia al vértice, eje de simetría, cortes con los ejes coordenados y concavidad.

Como función de proporción, la parábola toma interés desde el marco de las funciones exponenciales, específicamente como un caso de proporción directa donde si se supone constante entonces $y / r^{n}=k, y=k x^{*}$ luego, cuando $n=2$ la proporción expresada por $y=k x^{2}$ representa la manera como $x$ varía directamente con el cuadrado de $y$.

\section{Diseño de la experiencia y sistematización}

Como la propuesta pretendía establecer vínculos entre el acercamiento a realizar y el futuro desempeño profesional, se consideró como hilo conductor didáctico la propuesta de Sánchez (1996), quien a través de una serie de fases propone un trabajo centrado en los estudiantes y partiendo de la contextualización, los lleva hasta la aplicación. De igual manera, con miras a brindar una experiencia del mismo objeto matemático desde diferentes puntos de vista se consideró relevante el uso y paso por las diferentes representaciones.

A partir de estas consideraciones, la experiencia de aula se diseñó en tres fases: contextualización, construcción y ampliación. Para el acercamiento a las dos primeras etapas se propuso a los estudiantes una situación problema donde la parábola era abordada como lugar geométrico (representación geométrica), ya que les permitía un acercamiento constructivo.

- Contextualización. Esta fase a su vez, se desarrolló en dos etapas: la exploración, que tenía como objetivo motivar a los estudiantes en el comienzo del proceso de aprendizaje de la parábola, y la puesta en común, donde se permitía el planteamiento de conflictos cognitivos a partir de las diferentes opiniones presentadas por ellos.

- Construcción. Esta fase se llevó a cabo en tres etapas: elaboración de definiciones, cuyo objetivo era que los estudiantes comunicaran el resultado de sus discusiones ante la situación problema a partir de preconceptos; el razonamiento dirigido, que buscaba pasar la parábola de la representación geométrica y gráfica a la representación simbólica; y la generalización, donde se pretendía enfatizar en los invariantes de esta cónica.

- Ampliación o aplicaciones a la Ingeniería Civil. Esta fase estaba compuesta de tres etapas: la realización de investigación, cuyo objetivo era obtener la parábola como una sección plana mediante la intersección entre un plano y un cono de dos hojas; la resolución de problemas de aplicación, buscando mostrar la importancia de la parábola en una situación propia de la ingeniería civil; y el uso de contraejemplos, como camino para fortalecer la apropiación del objeto de estudio.

Para cada una de las fases se elaboró un diagrama de flujo de intervención como el que se muestra en la Figura 1. En la primera columna se señalan las 
los aspectos a evaluar y en la última, la forma de registro. De igual manera a través de flechas se indi- can las acciones a seguir resultado de la respuesta de los estudiantes.

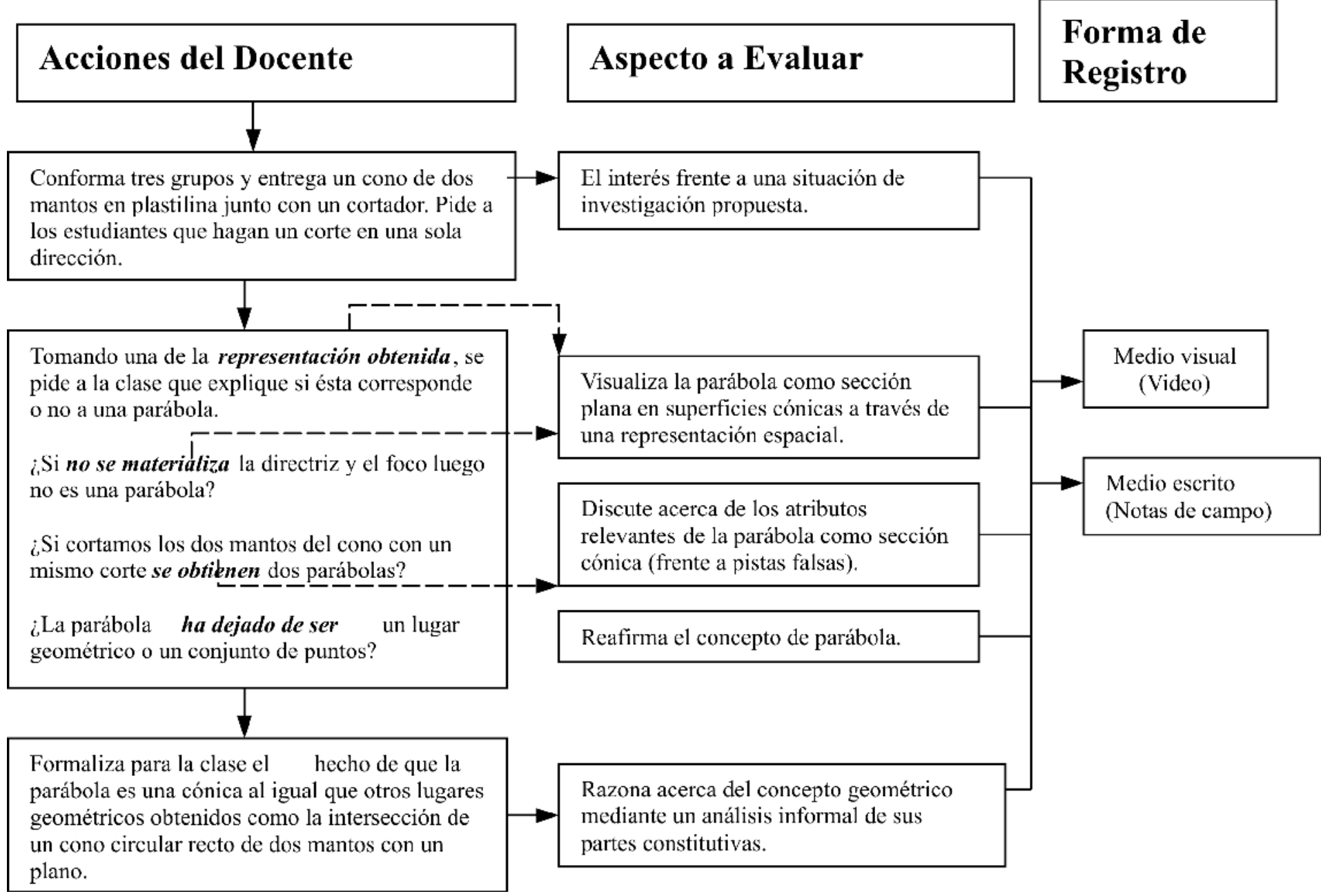

Figura 1. Ejemplo de diagrama de intervención en el aula. (Fase ampliación) Fuente: Los autores.

Para la obtención de los registros se utilizaron medios escritos y audiovisuales. En el caso de los escritos, se tuvieron en cuenta las notas de clase, el material elaborado por los estudiantes y el diario de campo del docente. Con ayudas audiovisuales se realizaron grabaciones de los estudiantes interactuando en sus grupos de trabajo y videos de cada una de las sesiones.

\section{Resultados.}

En el cuadro 1 se presenta una comparación entre las actividades programadas en las distintas fases y las desarrolladas. Cabe aclarar que el taller ${ }^{4}$ No. 4 "Descubriendo la cónica" surgió durante la etapa de experimentación ya que fue necesario presentar a los estudiantes un contra-ejemplo que permitiera diferenciar la parábola de la hipérbola.

Las tres sesiones se llevaron a cabo entre el 19 de Octubre y el 26 de Octubre en tres bloques de clase de 1 hora y 30 minutos que es el establecido en UNIMINUTO.

\footnotetext{
${ }^{4}$ Ver Anexo talleres utilizados durante las diferentes actividades.
} 


\begin{tabular}{|c|c|c|c|c|c|}
\hline \multirow{2}{*}{ FASE } & \multicolumn{2}{|c|}{ PLANEACIÓN } & \multicolumn{3}{|c|}{ EXPERIMENTACIÓN } \\
\hline & Etapas & Tiempo & Etapas & Tiempo & $\begin{array}{l}\text { Instrumento de } \\
\text { Registro }\end{array}$ \\
\hline Contextualización & $\begin{array}{c}\text { Taller 1: "Proyecto } \\
\text { Vía Panamerica- } \\
\text { na" }\end{array}$ & \multirow{4}{*}{$\begin{array}{c}1 \text { sesión } \\
(1 \mathrm{~h}-30 \mathrm{~min})\end{array}$} & $\begin{array}{c}\text { Taller 1: } \\
\text { "Proyecto Vía } \\
\text { Panamericana }\end{array}$ & \multirow{4}{*}{$\begin{array}{c}1 \text { sesión } \\
(1 h-45 \mathrm{~min})\end{array}$} & Video y Fotos \\
\hline \multirow{3}{*}{ Construcción } & $\begin{array}{l}\text { Elaboración de } \\
\text { una definición }\end{array}$ & & $\begin{array}{l}\text { Elaboración de } \\
\text { una definición }\end{array}$ & & $\begin{array}{c}\text { Video - Fotos y } \\
\text { acetatos }\end{array}$ \\
\hline & $\begin{array}{c}\text { De la representa- } \\
\text { ción geométrica } \\
\text { a la representa- } \\
\text { ción gráfica. }\end{array}$ & & $\begin{array}{l}\text { De la representa- } \\
\text { ción geométrica } \\
\text { a la representa- } \\
\text { ción gráfica. }\end{array}$ & & Video y Fotos \\
\hline & $\begin{array}{c}\text { De la represen- } \\
\text { tación gráfica a } \\
\text { la representación } \\
\text { simbólica }\end{array}$ & & $\begin{array}{l}\text { De la represen- } \\
\text { tación gráfica a } \\
\text { la representación } \\
\text { simbólica }\end{array}$ & & Video y Fotos \\
\hline \multirow{4}{*}{ Ampliación } & $\begin{array}{c}\text { Descubriendo la } \\
\text { parábola en un } \\
\text { cono }\end{array}$ & \multirow{4}{*}{$\begin{array}{c}1 \text { sesión } \\
(1 \mathrm{~h}-30 \mathrm{~min})\end{array}$} & $\begin{array}{l}\text { Descubriendo la } \\
\text { parábola en un } \\
\text { cono }\end{array}$ & \multirow{2}{*}{$\begin{array}{c}1 \text { sesión } \\
(1 \mathrm{~h}-45 \mathrm{~min})\end{array}$} & Video y Fotos \\
\hline & $\begin{array}{c}\text { Taller 2: } \\
\text { "Construyendo la } \\
\text { entrada al cielo" }\end{array}$ & & $\begin{array}{c}\text { Taller 2: } \\
\text { "Construyendo la } \\
\text { entrada al cielo" }\end{array}$ & & $\begin{array}{l}\text { Video - Fotos y } \\
\text { hojas de trabajo }\end{array}$ \\
\hline & \multirow{2}{*}{$\begin{array}{c}\text { Taller } 3 \\
\text { "Mejorando la } \\
\text { calidad de vida } \\
\text { de arborizadora } \\
\text { alta" }\end{array}$} & & $\begin{array}{c}\text { Taller } 3 \\
\text { "Mejorando la } \\
\text { calidad de vida } \\
\text { de arborizadora } \\
\text { alta" }\end{array}$ & \multirow[t]{2}{*}{$\begin{array}{c}1 \text { sesión } \\
(1 h-45 \mathrm{~min})\end{array}$} & Video \\
\hline & & & $\begin{array}{c}\text { Taller No. } 4 \\
\text { "Descubriendo la } \\
\text { Cónica" }\end{array}$ & & $\begin{array}{l}\text { Video y hojas de } \\
\text { trabajo. }\end{array}$ \\
\hline
\end{tabular}

Cuadro 1. Comparación entre las actividades planteadas y las experimentadas. Fuente: Los autores

Durante la fase de contextualización, el uso de una carta (Taller 1 "Construcción de la vía panamericana"), cuyo fin era una contratación para participar en un proyecto vial resultó ser una estrategia llamativa para los estudiantes. Al enfrentar el problema inmediatamente buscaron en sus apuntes y en textos que tenían a su alcance la solución, abandonando la posibilidad de utilizar una serie de elementos de medida que el docente había dispuesto para la actividad (compás, reglas y cuerdas). Frente a esto, el docente se vió abocado a insistir a los estudiantes que respondieran la carta y usaran los materiales. Con ello, después de medir con los diferentes elementos la distancia entre puntos, se generó un ambiente de discusión entre alumnos y docente que favoreció la emergencia de preconceptos como: distancia entre dos puntos, distancia entre un punto y una recta, y rectas perpendiculares. Finalmente, en el espacio de la puesta en común, ellos justificaron los diferentes procedimientos construidos para llegar a su conjetura, haciendo uso de un lenguaje informal y dejando entre ver aspectos que serían relevantes en la siguiente etapa. Ver Figura 2.
En la fase de construcción, el docente listó en el tablero los invariantes ${ }^{5}$ identificados por los estudiantes y los invitó a elaborar una definición de parábola donde se evidenciaron dificultades para escribir de manera clara y precisa sus ideas. Luego, para pasar de la representación geométrica a la gráfica (plano cartesiano) el docente presentó nuevamente la situación problema pero ya en el marco de coordenadas geográficas, lo cual generó mucho más interés y sentido frente al uso de la parábola en el diseño de vías en un contexto real. Ver Figura 2.

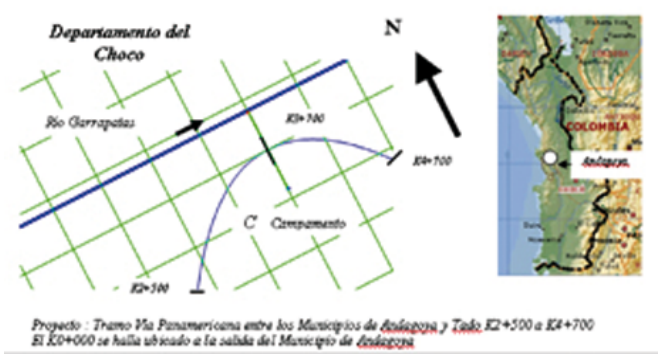

Figura 2. Representación gráfica de la situación problema. Fuente: Los autores 
Durante la etapa de ampliación, los estudiantes lograron identificar la parábola, la circunferencia y la hipérbola como el resultado de diferentes cortes sobre un cono de dos hojas elaborado por ellos mismo en plastilina. Luego, al proponer el Taller 2 "Construyendo la entrada al cielo", donde se pedía el diseño de una puerta de entrada a una iglesia con forma parabólica, la actitud fue de creatividad y uso de diferentes materiales en la construcción de su modelo en papel. Al pedirles que escribieran una manera de demostrar que la puerta por ellos diseñada y construida era realmente una parábola, surgieron propuestas muy interesantes que evidenciaron la importancia que para los estudiantes había tomado el invariante de la parábola.

En esta fase, se propuso el Taller 3 "Mejorando la calidad de vida de Arborizadora Alta" en el cual se solicitaba diseñar un pozo séptico para la disposición de aguas residuales en una vivienda de interés social. Al enfrentar la situación, los estudiantes siguieron caminos no solamente gráficos y geométricos, sino también algebraicos buscando modelar la trayectoria del chorro de llegada al tanque con una función cuadrática e inclusive utilizaron ecuaciones propias del estudio del movimiento parabólico para resolver la situación.

\section{Análisis de la comprensión}

Entendiendo razonar en geometría como "comprender los distintos elementos que conforman una teoría geométrica.... y haciendo referencia a acciones como manipular e interpretar sintáctica y semánticamente diversos representaciones de conceptos y procedimientos y establecer relaciones entre estos" (Samper, Camargo y Leguizamón, 2001), durante las distintas fases se encontró que:

a) A la hora de conjeturar sobre la situación problema los estudiantes utilizaron primero el razonamiento visual, los preconceptos y sus experiencias o gustos personales. Luego, a la hora de argumentar sus primeras soluciones al problema, emplearon un razonamiento empírico ya que hacían generalizaciones a partir de dos o tres casos y apoyados en el uso de la medida como demostración.

b) El uso de la ubicación geográfica de la vía en forma parabólica fue fundamental para que pasaran de la representación geométrica a la gráfica. De igual manera, la equidistancia punto-vértice y punto-directriz fue importante a la hora de pasar al

${ }^{5}$ Un invariante es algo que no cambia al aplicarle un conjunto de transformaciones. La propiedad de ser invariante se conoce como invarianza. (Joya, 2007) lenguaje algebraico y llegar a la expresión estándar y general de una parábola, como se muestra en la figura 3. Una vez lograda la representación algebraica los estudiantes lograron la expresión de la parábola para el caso en que se considera una traslación de ejes.

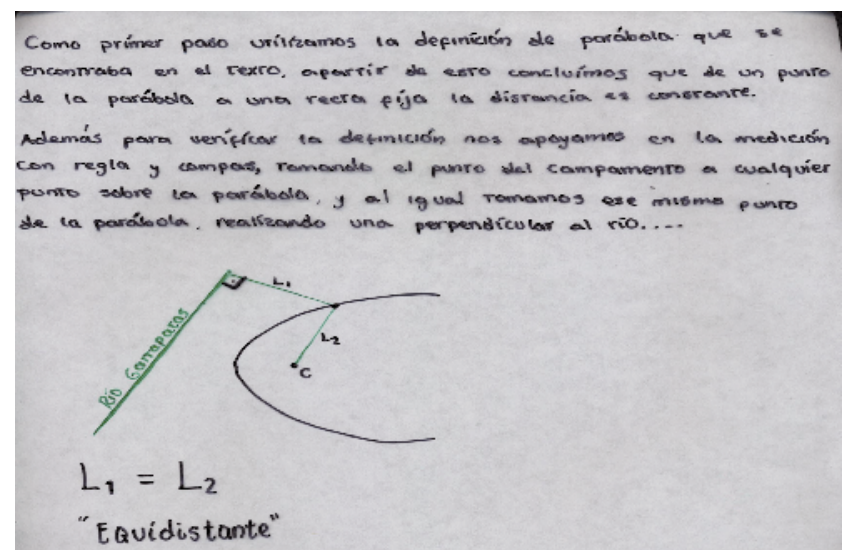

Figura 3. Producción del Grupo No. 3 frente al Taller No. 1. Fuente: Los autores

c)Durante la fase de aplicación, específicamente frente al Taller 2, cuando los estudiantes diseñaron la puerta en forma parabólica, se constató que los elementos más importantes en su construcción eran: foco, directriz, vértice y el eje de simetría. De otra parte para ubicar otros puntos sobre el plano y poder trazar la parábola le dieron relevancia a acciones como que los puntos deberían estar sobre rectas que fueran perpendiculares a la directriz y luego garantizaban la equidistancia por ensayo y error mediante la medición con diferentes instrumentos y el uso de la reflexión de cada punto a partir del eje de simetría.

Frente al Taller No 3, luego de identificar el chorro de agua en el interior del pozo séptico como media parábola, identificaron los siguientes elementos: el eje de simetría (la pared del tanque), el vértice (punto de salida del chorro) y un punto de la parábola del cual se daba su ubicación $(x, y)$ en el ejercicio. Luego utilizando la ecuación de la parábola de la forma $x^{2}=4 p y$ para $p<0$, y considerando un punto sobre esta de coordenadas $(3 m, 2.4 m)$ s estudiantes calcularon el valor del parámetro $p$. Finalmente, utilizaron la ecuación determinada para hallar el valor de $x$ dado un $y=7.5 \mathrm{~m}$ que representa la profundidad del tanque.

El Taller 4, "Descubriendo la cónica", enfrentó a los estudiantes a afirmaciones hechas por ellos durante las sesiones 1 y 2 en particular "una hipérbola está compuesta por dos parábolas enfrentadas". En este caso, todos los grupos utilizaron como método de explicación la medida con ayuda de una regla, ha- 
llando los valores que les permitían decidir si era una hipérbola o una parábola.

\section{Análisis de la interacción didáctica}

Respecto a la gestión en el aula, se estableció que iniciar con una situación problema es válido porque motiva a los estudiantes frente a una situación real y logra en ellos un ambiente de participación. Las acciones llevadas a cabo durante la fase de construcción (descubrimiento dirigido) fueron pertinentes ya que se respetaron al máximo sus producciones verbales y escritas, donde ellos fueron los que determinaron qué era cierto y qué no.

La fase de ampliación (aplicaciones a la ingeniería) resultó ser la más motivadora. Los talleres 2 y 3 estaban al alcance de sus capacidades y conocimientos, despertando en ellos gran curiosidad e interés. El docente contribuyó incentivándolos a participar activamente, escuchando sus propuestas y argumentos, respetando los diferentes ritmos de aprendizaje y favoreciendo las vivencias de éxito. La situación planteada en la última sesión, permitió reconocer la fuerza que tienen los contra-ejemplos para justificar la falsedad de una afirmación dada, a pesar de que no fue una situación incluida en la etapa de planeación.

Frente al tratamiento del contenido matemático en juego, se puede afirmar que el paso por las diferentes representaciones fue evidente a lo largo de las sesiones realizadas. En algunas ocasiones ésta situación fue inducida por los profesores y en otras los estudiantes se sintieron con los elementos suficientes y toda la libertad para hacerlo.

A medida que se desarrollaron las actividades, utilizaron algunos elementos relevantes de la parábola como lugar geométrico de los cuales nunca se les habló y que se convirtieron en aspectos claves para enfrentar las diferentes fases:

-El eje de simetría, elemento fundamental a la hora de elaborar construcciones y desarrollar simetría axial.

- El vértice, se transformó en la guía para construir otros puntos de la parábola, incluso lo titularon como "punto inicial" en las construcciones en papel. - La perpendicularidad de la distancia de cualquier punto de la parábola a la directriz.

\section{Conclusiones}

- Para introducir la parábola a estudiantes de ingeniería civil, es pertinente iniciar con su planteamiento dentro de una situación problema, en contextos propios de la disciplina, ya que de esta manera se motiva y se destaca la importancia de la cónica.
Se recomienda iniciar el estudio de la parábola utilizando las representaciones: geométrica, gráfica y verbal.

- De acuerdo al desempeño de los estudiantes en los talleres planteados, se afirma que los elementos relevantes de la parábola fueron identificados por ellos y utilizados a la hora de solucionar situaciones propias de la ingeniería civil.

- Se sugiere plantear actividades que giren alrededor de la visualización y el razonamiento discursivo. Es importante que los estudiantes desarrollen capacidad de abstracción y justificación frente a problemas reales.

-Es necesario abordar la enseñanza de la parábola en ingeniería por medio de la geometría dinámica apoyada en las TIC, ya que en papel y lápiz las tareas de descubrimiento y aplicación resultan ser bastante demoradas, lo cual limita el análisis de otros aspectos.

- De acuerdo al trabajo realizado en la etapa de construcción, es necesario asumir la enseñanza de la parábola desde la representación geométrica en estudiantes de educación media.

\section{Referencias.}

[1] Azcarate, C. (2001) Definiciones, demostraciones ¿Por qué?, ¿Cuándo?, ¿Cómo? Memorias Novena Jornada para el Aprendizaje y la Enseñanza de las Matemáticas. Barcelona España.

[2] Bruño, G. (1960). Geometría Curso Superior. Procuraduría General de Paris. Francia

[3] Camargo, L., Leguizamón, C. \& Samper, C. (2001). Razonamiento en Geometría, Revista Educación Matemática. Vol 6, . Número 2. Julio.

[4[ Camargo, L. \& Guzman, A. (2001). Comprensión de las Relaciones entre Pendiente y la Razón de Cambio. Trabajo de Grado de Maestría en Docencia de la Matemática Universidad Pedagógica Nacional. Bogotá D.C.

[5] De Villiers, M. (1993). El Papel de la Demostración y Función en Matemáticas. Revista Epsilon. Número 26. [6] Font, V. (2001). Reflexiones Didácticas desde y para el aula expresiones simbólicas a partir de gráficas en el caso de la parábola. Revista Educación Matemática. Vol 6, Número 2.

[7] Joya, A (2007). Nuevas Matemáticas 10. Editorial Santillana. Bogotá D.C

[8] Lehman, Ch. (1996). Geometría Analítica. Editorial Limusa. Madrid, España.

[9] Kruglak, H. \& Moore, J. (1981) Teoría y Problemas de Matemáticas Aplicadas a Ciencia y Tecnología. Editorial Mac-Graw Hill. México 
[10] Sánchez, J. (1996) Lugares Geométricos Cónicas. Editorial Síntesis. Madrid, España.

[1 1] Stewart, J.(2001) Cálculo de una Variable. Editorial Thomson - Learning. México.
[12] Thomas, G., \& Finney, R. (1999). Cálculo Una Variable. Editorial Pearson Educación. México.

[13] UNIMINUTO (2001). Modelo Educativo. Publicación institucional V. 2.0. Bogotá D.C

Sara Giraldo J. M. Sc en Didáctica de las Matemáticas de la Universidad Pedagógica Nacional. M. Sc en Matemáticas de la Universidad Nacional sede Bogotá. Especialista en Educación Matemática de la Universidad Pedagógica Nacional. Especialista en Análisis de Datos de la Universidad de la Salle de Bogotá. Licenciada en Educación Matemática de la Universidad Pedagógica Nacional. Actualmente es la Directora del Departamento de Ciencias Básicas, Facultad de Ingeniería, Corporación Universitaria Minuto de Dios, UNIMINUTO, Sede principal, Bogotá D.C. sgiraldo@uniminuto.edu

Frey Rodríguez P. Especialista en Educación Matemática de la Universidad Pedagógica Nacional. Ingeniero Agrícola de la Universidad Nacional sede Bogotá. Aspirante al titulo de M.Sc en Informática Educativa de la Universidad de la Sabana. Docente de tiempo completo del Departamento de Ciencias Básicas, Facultad de Ingeniería, Corporación Universitaria Minuto de Dios, UNIMINUTO, Sede principal, Bogotá D.C.

frodriguez@uniminuto.edu 